\title{
A Dynamic Programming Approach to Price Installment Options*
}

\author{
Hatem Ben-Ameur ${ }^{\dagger} \quad$ Michèle Breton ${ }^{\dagger} \quad$ Pascal François ${ }^{\ddagger}$
}

April 2004

${ }^{*}$ Corresponding author: Michèle Breton, Centre for Research on e-finance, 3000 CôteSte-Catherine, Montreal H3T 2A7, Canada. Mail to: michele.breton@hec.ca.

${ }^{\dagger}$ CREF, GERAD and HEC Montréal

${ }^{\ddagger} \mathrm{CREF}$ and HEC Montréal. 


\title{
A Dynamic Programming Approach to Price Installment Options
}

\begin{abstract}
Installment options are Bermudan-style options where the holder periodically decides whether to exercise or not and then to keep the option alive or not (by paying the installment). We develop a dynamic programming procedure to price installment options. We study in particular the Geometric Brownian Motion case and derive some theoretical properties of the IO contract within this framework. We also characterize the range of installments within which the installment option is not redundant with the European contract. Numerical experiments show the method yields monotonically converging prices, and satisfactory trade-offs between accuracy and computational time. Our approach is finally applied to installment warrants, which are actively traded on the Australian Stock Exchange. Numerical investigation shows the various capital dilution effects resulting from different installment warrant designs.
\end{abstract}

Keywords: Finance, Dynamic Programming, Option pricing, Installment Option, Installment Warrant.

Acknowledgements: We acknowledge financial support from NSERC, SSHRC, IFM ${ }^{2}$ and HEC Montréal. 


\section{Introduction}

Installment Options (IO) are akin to Bermudan options except that the holder must regularly pay a premium (the "installment") to keep the option alive. The pre-specified dates (thereafter "decision dates") at which the IO may be striked correspond to the installment schedule. Therefore, at each decision date, the holder of the IO must choose between the following

1. to exercise the option, which puts an end to the contract;

2. not to exercise the option and to pay the installment, which keeps the option alive until the next decision date;

3. not to exercise the option and not to pay the installment, which puts an end to the contract.

Among the most actively traded installment options throughout the world presently are the installment warrants on Australian stocks listed on the Australian Stock Exchange (ASX). Installment options are a recent financial innovation that introduces some flexibility in the liquidity management of portfolio strategies. Instead of paying a lump sum for a derivative instrument, the holder of the IO will pay the installments as long as the need for being long in the option is present. In particular, this considerably reduces the cost of entering into a hedging strategy. ${ }^{1}$ In addition, the

\footnotetext{
${ }^{1}$ Risk managers may enter the IO contract at a low initial cost and adjust the installment schedule with respect to their cash forecasts and liquidity constraints. This feature is particularly attractive for corporations which massively hedge interest rate and currency risks with forwards, futures or swaps because standard option contracts imply a cost at entry that may be incompatible with a temporary cash shortage.
} 
non-payment of an installment suffices to close the position at no transaction cost. This reduces the liquidity risk typically associated with other over-the-counter derivatives.

The aim of this paper is twofold. First, we tackle the problem of pricing IOs using Dynamic Programming (DP) in a general setting. Second, we investigate the properties of IOs through theoretical and numerical analysis in the Black and Scholes (1973) setting.

Literature on IOs is scarce. Davis, Schachermayer and Tompkins (2001, 2002) derive no-arbitrage bounds for the price of the IO and study static versus dynamic hedging strategies within a Black-Scholes framework with stochastic volatility. Their analysis however is restricted to European-style IOs, which allows for an analogy with compound options. Davis, Schachermayer and Tompkins (2003) value venture capital using an analogy with IO.

Algorithms based on finite differences have been widely used for pricing options with no known closed-form solution (see e.g. Wilmott, Dewynne and Howison (1993) for a survey). Dynamic programming stands as an alternative for low dimensional option pricing. By contrast to finite difference methods, DP does not require time discretization. A DP formulation for pricing American options can be traced back to Chen (1970). He was able to generate theoretical prices directly for a limited number of decision dates. Note however that his paper appeared before the seminal Black and Scholes (1973) contribution and therefore does not apply risk neutral pricing.

Ben Ameur, Breton and L'Écuyer (2002) show that DP combined with finite elements is particularly well suited for options involving decisions at a limited number of distant dates during the life of the contract. Examples include Bermudan-style options, callables, and convertibles. By construc- 
tion, IOs allow for both early exercise and installment payment decisions periodically.

The rest of the paper is organized as follows. In Section 2, we develop the model. In Section 3, we solve the Bellman equation and show how the discretization and approximation are made. Section 4 presents the special case of the Geometric Brownian Motion and derives properties of the value function in this setting. We present numerical illustrations in Section 5. In Section 6 we show how to adapt our approach to the pricing of installment warrants, which are actively traded on the Australian Stock Exchange (ASX). Section 7 concludes.

\section{The model}

Let the price of the underlying asset $\{S\}$ be a Markov process that verifies the fundamental no-arbitrage property. Let $t_{0}=0$ be the installment option (IO) inception date and $t_{1}, t_{2}, \ldots, t_{n}\left(t_{n}=T\right)$ a collection of decision dates scheduled in the contract. An installment design is characterized by the vector of premia $\pi=\left(\pi_{1}, \ldots, \pi_{n-1}\right)$ that are to be paid by the holder at dates $t_{1}, \ldots, t_{n-1}$ to keep the $\mathrm{IO}$ alive. The price of the $\mathrm{IO}$ is the upfront payment $v_{0}$ required at $t_{0}$ to enter the contract.

The exercise value of the IO at any decision date $t_{m}$, for $m=1, \ldots, n$, is explicit in the contract and given by

$$
v^{e}(s)= \begin{cases}(s-K)^{+}, & \text {for the installment call option } \\ (K-s)^{+}, & \text {for the installment put option }\end{cases}
$$

where $s=S_{t_{m}}$ is the price of the underlying asset at $t_{m}$ and $(x)^{+}$denotes the function $\max \{0, x\}$. By the risk-neutral principle, the holding value of 
the option at $t_{m}$ is

$$
v_{m}^{h}(s)=E\left[\rho(m) v_{m+1}\left(S_{t_{m+1}}\right) \mid S_{t_{m}}=s\right], \quad \text { for } m=0, \ldots, n-1,
$$

where

$$
v_{m}(s)=\left\{\begin{array}{l}
v_{0}^{h}(s) \quad \text { for } m=0 \\
\max \left(v^{e}(s), v_{m}^{h}(s)-\pi_{m}\right) \quad \text { for } m=1, \ldots, n-1 \\
v^{e}(s) \quad \text { for } m=n
\end{array}\right.
$$

and

$$
\rho(m)=e^{-\int_{t_{m}}^{t_{m+1}} r(u) d u}
$$

is the discount factor.

The function $v_{m}^{h}(s)-\pi_{m}$ is called thereafter the net holding value at $t_{m}$, for $m=1, \ldots, n-1$ and the function $v_{m}$ is the value function representing the value of the IO at date $t_{m}$ as a function of the price of the underlying asset. Equation (3) models the choices that are available to the option holder: he will pay the installment and hold the option as long as the net holding value is larger than the exercise value. Otherwise, according to the exercise value, he will either exercise the option (when positive) or abandon the contract (when null).

One way of pricing this IO is via backward induction using (1)-(3) from the known function $v_{n}=v^{e}$. However, the value function $v_{m}$, for $m=$ $0, \ldots, n-1$, is generally not known analytically and must be approximated in some way. In the following section, we propose an approximation over a finite grid. 


\section{Solving the DP equation}

In this section, we compute the expectation in (2). The idea is to partition the positive real axis into a collection of intervals and then to approximate the option value by a piecewise linear interpolation.

Let $a_{0}=0<a_{1}<\ldots<a_{p}<a_{p+1}=+\infty$ be a set of points partitioning the positive real line into $(p+1)$ intervals

$$
\left(a_{i}, a_{i+1}\right] \quad \text { for } i=0, \ldots, p \text {. }
$$

Given an approximation $\widetilde{v}_{m}$ of the option value $v_{m}$ at the discrete points $a_{i}$ and step $m$, this function is interpolated piecewise linearly, which yields

$$
\widehat{v}_{m}(s)=\sum_{i=0}^{p}\left(\alpha_{i}^{m}+\beta_{i}^{m} s\right) I\left(a_{i}<s \leq a_{i+1}\right),
$$

where $I$ is the indicator function. The local coefficients of this interpolation at step $m$, that is the $\alpha_{i}^{m}$ 's and the $\beta_{i}^{m}$ 's, are obtained by solving the linear equations

$$
\widetilde{v}_{m}\left(a_{i}\right)=\widehat{v}_{m}\left(a_{i}\right), \quad \text { for } i=0, \ldots, p-1,
$$

that is:

$$
\begin{aligned}
\alpha_{i}^{m} & =\frac{\widetilde{v}_{m}\left(a_{i}\right) a_{i+1}-\widetilde{v}_{m}\left(a_{i+1}\right) a_{i}}{a_{i+1}-a_{i}} \\
\beta_{i}^{m} & =\frac{\widetilde{v}_{m}\left(a_{i+1)}-\widetilde{v}_{m}\left(a_{i}\right)\right.}{a_{i+1}-a_{i}}
\end{aligned}
$$

For $i=p$, we take

$$
\alpha_{p}^{m}=\alpha_{p-1}^{m} \text { and } \beta_{p}^{m}=\beta_{p-1}^{m}
$$


Assume now that $\widehat{v}_{m+1}$ is known. The expectation in (2) at step $m$ becomes

$$
\begin{aligned}
& \widetilde{v}_{m}^{h}\left(a_{k}\right) \\
= & E\left[\rho(m) \widehat{v}_{m+1}\left(S_{t_{m+1}}\right) \mid S_{t_{m}}=a_{k}\right] \\
= & \rho(m) \sum_{i=0}^{p} \alpha_{i}^{m+1} E\left[I\left(a_{i}<S_{t_{m+1}} \leq a_{i+1}\right) \mid S_{t_{m}}=a_{k}\right] \\
& +\beta_{i}^{m+1} E\left[S_{t_{m+1}} I\left(a_{i}<S_{t_{m+1}} \leq a_{i+1}\right) \mid S_{t_{m}}=a_{k}\right],
\end{aligned}
$$

where $\widetilde{v}_{m}^{h}$ denotes the approximate holding value of the IO. For $k=1, \ldots, p$ and $i=0, \ldots, p$, denote

$$
\begin{aligned}
A_{k i}^{m} & =E\left[I\left(a_{i}<S_{t_{m+1}} \leq a_{i+1}\right) \mid S_{t_{m}}=a_{k}\right] \\
B_{k i}^{m} & =E\left[S_{t_{m+1}} I\left(a_{i}<S_{t_{m+1}} \leq a_{i+1}\right) \mid S_{t_{m}}=a_{k}\right],
\end{aligned}
$$

so that

$$
\widetilde{v}_{m}^{h}\left(a_{k}\right)=\rho(m) \sum_{i=0}^{p} \alpha_{i}^{m+1} A_{k i}^{m}+\beta_{i}^{m+1} B_{k i}^{m} .
$$

Key in the applicability of the DP procedure is how efficiently the integrals (9)-(10) can be computed. Three cases are of special interest. The expectations (9) and (10) can be computed:

1. In closed-form (e.g. the Geometric Brownian Motion, the OrnsteinUhlenbeck process, the square-root process, Garch),

2. By numerical integration (e.g. CEV processes),

3. By Monte Carlo simulations (e.g. stochastic volatility models).

Note that expectations (9) and (10) can be related to the price of digital options within $\left[t_{m}, t_{m+1}\right]$. Also note that in case 3 , the efficiency of the computation of expectations (9) and (10) decreases dramatically as $\left[a_{i}, a_{i+1}\right]$ 
moves away from $a_{k}$. This is the well known problem of estimating the probability of rare events. Consequently, our approach is best suited for cases 1 and 2 .

The Dynamic Programming algorithm to price the IO may be summarized as follows (recall that $v^{n}$ is known):

1. Initialization: Set $\widetilde{v}^{n}\left(a_{k}\right)=v^{n}\left(a_{k}\right)$ for all $k$; set $m=n$;

2. Compute $\alpha_{i}^{m}$ and $\beta_{i}^{m}$ for $i=0, \ldots, p$ using (5-7);

3. If $m=0$, stop; the value of the IO is approximated by the piecewise linear function $\widehat{v}_{0}(\cdot)$; Otherwise, set $m=m-1$;

4. Compute $\widetilde{v}_{m}^{h}\left(a_{k}\right)$ for all $k$ using (11);

5. Compute $\widetilde{v}_{m}\left(a_{k}\right)$ for all $k$ using (3); record the optimal decision at $(m, k)$;

6. Go to step 2.

As an illustration, we show how our methodology applies to the Geometric Brownian Motion case. We also derive some theoretical properties of the IO contract within this framework.

\section{The Geometric Brownian Motion framework}

In that case, the price of the underlying asset satisfies

$$
\frac{d S_{t}}{S_{t}}=(r-\delta) d t+\sigma d W_{t}, \quad \text { for } 0 \leq t \leq T
$$


where $r$ is the riskless rate, $\delta$ is a constant dividend rate, $\sigma$ is the volatility of the return on the underlying asset, and $\{W\}$ is a standard Brownian motion.

The solution to this SDE verifies

$$
S_{t^{\prime \prime}}=S_{t^{\prime}} \exp (\mu \Delta t+\sigma \sqrt{\Delta t} Z), \text { for } 0 \leq t^{\prime} \leq t^{\prime \prime} \leq T,
$$

where $\mu=\left(r-\delta-\sigma^{2} / 2\right), \Delta t=t^{\prime \prime}-t^{\prime}$ and $Z$ is a standard normal random variable independent of the past of $\{S\}$ up to time $t^{\prime}$. Hence, denoting $\Delta t_{m} \equiv t_{m+1}-t_{m},(9)-(10)$ can be written:

$$
\begin{gathered}
A_{k i}^{m}=E\left[I\left(\frac{a_{i}}{a_{k}}<e^{\mu \Delta t_{m}+\sigma \sqrt{\Delta t_{m}} Z} \leq \frac{a_{i+1}}{a_{k}}\right)\right] \\
= \begin{cases}\Phi\left(c_{k, 1}\right) & \text { for } i=0 \\
\Phi\left(c_{k, i+1}\right)-\Phi\left(c_{k, i}\right) & \text { for } 1 \leq i \leq p-1 \\
1-\Phi\left(c_{k, p}\right) & \text { for } i=p\end{cases} \\
B_{k i}^{m}=E\left[\begin{array}{ll}
\left.a_{k} e^{\mu \Delta t_{m}+\sigma \sqrt{\Delta t_{m}} Z} I\left(\frac{a_{i}}{a_{k}}<e^{\mu \Delta t_{m}+\sigma \sqrt{\Delta t_{m}} Z} \leq \frac{a_{i+1}}{a_{k}}\right)\right] \\
a_{k} \Phi\left(c_{k, 1}-\sigma \sqrt{\Delta t_{m}}\right) e^{(r-\delta) \Delta t_{m}} & \text { for } i=0 \\
a_{k}\left[\Phi\left(c_{k, i+1}-\sigma \sqrt{\Delta t_{m}}\right)-\Phi\left(c_{k, i}-\sigma \sqrt{\Delta t_{m}}\right)\right] e^{(r-\delta) \Delta t_{m}} & \text { for } 1 \leq i \leq p-1, \\
a_{k}\left[1-\Phi\left(c_{k, p}-\sigma \sqrt{\Delta t_{m}}\right)\right] e^{(r-\delta) \Delta t_{m}} & \text { for } i=p
\end{array}\right.
\end{gathered}
$$

where $c_{k, i}=\left[\ln \left(a_{i} / a_{k}\right)-\mu \Delta t_{m}\right] /\left(\sigma \sqrt{\Delta t_{m}}\right)$, and $\Phi$ stands for the cumulative density function of $Z$.

We now derive some theoretical properties related to the design of installment call options in the GBM framework. Symmetric results hold for installment put options. To simplify the exposition and without loss of generality, from now on we will assume that all decision dates are equally spaced, with $\Delta t_{m}=\left(t_{m+1}-t_{m}\right)=\Delta t, m=0, \ldots n-1$. This also implies that $A_{k i}^{m}=A_{k i}, B_{k i}^{m}=B_{k i}$, and $\rho(m)=e^{-r \Delta t}$. 
Proposition 1 The net holding value of the IO call at $t_{m}, v_{m}^{h}(s)-\pi_{m}$, as a function of $s>0$, is continuous, differentiable, convex, and monotone with a positive rate less than 1. The value function is null on the exit region $\left(0, x_{m}\right)$, equal to the net holding value on the holding region $\left[x_{m}, y_{m}\right]$, and equal to the exercise value on the exercise region $\left(y_{m}, \infty\right)$ where $x_{m}$ and $y_{m}$ are two thresholds that depend on the IO parameters.

Proof. The proof proceeds by induction on $m=n-1, \ldots, 0$. At $t_{n-1}$, the holding value at $s>0$ is

$$
\begin{aligned}
v_{n-1}^{h}(s) & =e^{-r \Delta t} E\left[v_{n}\left(S_{t_{n}}\right) \mid S_{t_{n-1}}=s\right] \\
& =e^{-r \Delta t} \int_{-\infty}^{+\infty}\left(s e^{\mu \Delta t+\sigma \sqrt{\Delta t} z}-K\right)^{+} \phi(z) d z
\end{aligned}
$$

where $\phi$ is the density function of the standard normal distribution. Obviously, this function is always strictly positive. By the Lebesgue's dominated convergence theorem (Billingsley, 1995), the holding value is continuous, differentiable for all $s>0$, and

$$
\lim _{s \longrightarrow 0} v_{n-1}^{h}(s)=0
$$

Since $(k s-K)^{+}$is convex, $v_{n-1}^{h}$ is a convex function of $s>0$ as a convex combination of convex functions of $s>0$. It is monotone as an integral of a non decreasing function indexed by $s>0$. For $s_{2}>s_{1}>0$, one has

$$
\begin{aligned}
& v_{n-1}^{h}\left(s_{2}\right)-v_{n-1}^{h}\left(s_{1}\right) \\
= & e^{-r \Delta t} \int_{-\infty}^{+\infty}\left(\left(s_{2} e^{\mu \Delta t+\sigma \sqrt{\Delta t} z}-K\right)^{+}-\left(s_{1} e^{\mu \Delta t+\sigma \sqrt{\Delta t} z}-K\right)^{+}\right) \phi(z) d z \\
\leq & \left(s_{2}-s_{1}\right) e^{-\left(\delta+\sigma^{2} / 2\right) \Delta t} \int_{-\infty}^{+\infty} e^{\sigma \sqrt{\Delta t} z} \phi(z) d z \\
\leq & s_{2}-s_{1} .
\end{aligned}
$$


The increasing rate of the holding value at $t_{n-1}$ is thus less than 1 . Consequently, the net holding value reaches 0 at a unique threshold $x_{n-1}$, and the exercise value at a unique threshold $y_{n-1}$, where $x_{n-1}$ and $y_{n-1}$ depend on the IO parameters. Properties of the value function $v_{n-1}$ follow (see Figure 1 with $m=n-1)$. Now, if one assumes that these properties hold at step $m+1$, the same arguments may be used to prove that they hold at step $m$ (we omit the details).

Figure 1 plots the curve representing the net holding value of the installment call option $v_{m}^{h}(s)-\pi_{m}$ for any decision date $m$. This curve intersects the $x$-axis at $x_{m}$ which separates the exit region from the holding region. Since its slope is less than 1 , it necessarily intersects the call intrinsic value at $y_{m}$, which separates the holding region from the exercise region.

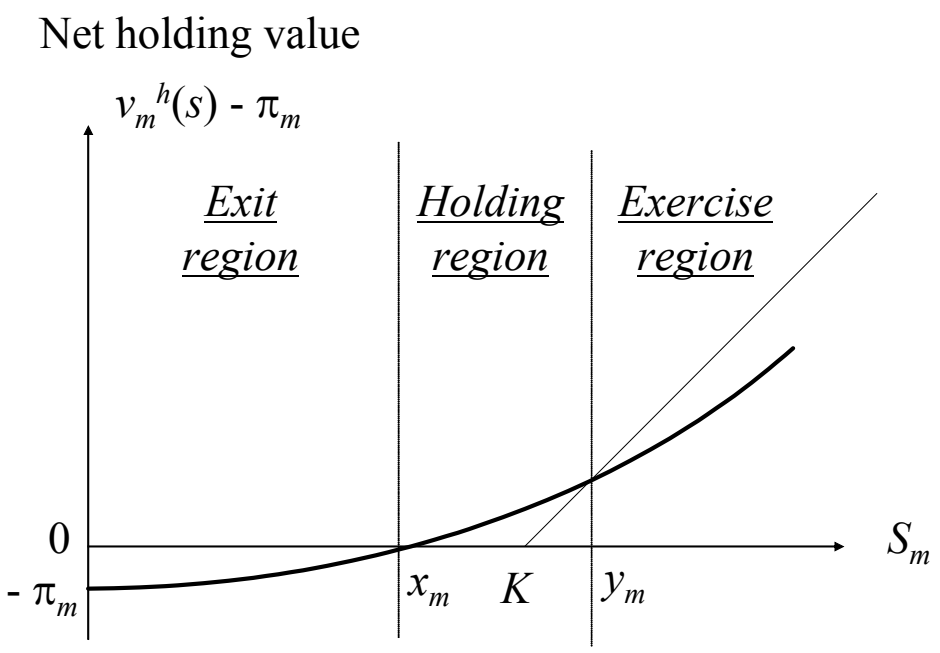

Figure 1

Theoretical properties of the value function can be used in the DP algorithm to speed up the computations. Indeed, as soon as the holding region 
$\left[x_{m}, y_{m}\right]$ is obtained, the evaluation of the value function in step 5 and of its piecewise linear approximation in step 4 are straightforward for values of $s$ outside $\left[x_{m}, y_{m}\right]$.

Let $C(s, \sigma, K, T, r, \delta)$ denote the price of the European call option with current price $s$, volatility $\sigma$, strike $K$, maturity $T$, interest rate $r$ and dividend rate $\delta$. The following Proposition establishes conditions on the premia so that the holding region vanishes.

Proposition 2 For $k=1, \ldots, n-1$, assume that $\pi_{m} \geq C(K, \sigma, K, \Delta t, r, \delta)$, for all $m \geq k$. Then one has

$$
v_{k}(s)=v^{e}(s), \text { for all } s>0 .
$$

Proof. The proof is established by induction on $m=n-1, \ldots, 1$. At time $t_{n-1}$, one has

$$
\begin{aligned}
v_{n-1}^{h}(K) & =E\left[e^{-r \Delta t} v_{n}\left(S_{t_{n}}\right) \mid S_{t_{n-1}}=K\right] \\
& =E\left[e^{-r \Delta t} v^{e}\left(S_{t_{n}}\right) \mid S_{t_{n-1}}=K\right] \\
& =C(K, \sigma, K, \Delta t, r, \delta) .
\end{aligned}
$$

Recall that the holding value of a call IO is a monotone function of $s>0$ with a positive rate less than 1 (see Proposition 1). As a consequence, for $\pi_{n-1} \geq C(K, \sigma, K, \Delta t, r, \delta)$, the net holding value at $t_{n-1}, v_{n-1}^{h}(s)-\pi_{n-1}$, is always lower than the exercise value, $v^{e}(s)$ (see Figure 2).

At step $k+1$, assume that $\pi_{m} \geq C(K, \sigma, K, \Delta t, r, \delta)$ and $v_{m}(s)=v^{e}(s)$, for all $s>0$ and $m \geq k+1$. One has

$$
\begin{aligned}
v_{k}^{h}(K) & =E\left[e^{-r \Delta t} v_{k+1}\left(S_{t_{k+1}}\right) \mid S_{t_{k}}=s\right] \\
& =E\left[e^{-r \Delta t} v^{e}\left(S_{t_{k+1}}\right) \mid S_{t_{k}}=s\right] \\
& =C(K, \sigma, K, \Delta t, r, \delta) .
\end{aligned}
$$


Again, since $v_{k}^{h}$ is monotone with a positive rate less than 1 , this implies

$$
v_{k}(s)=v^{e}(s) \quad \text { for all } s>0 .
$$

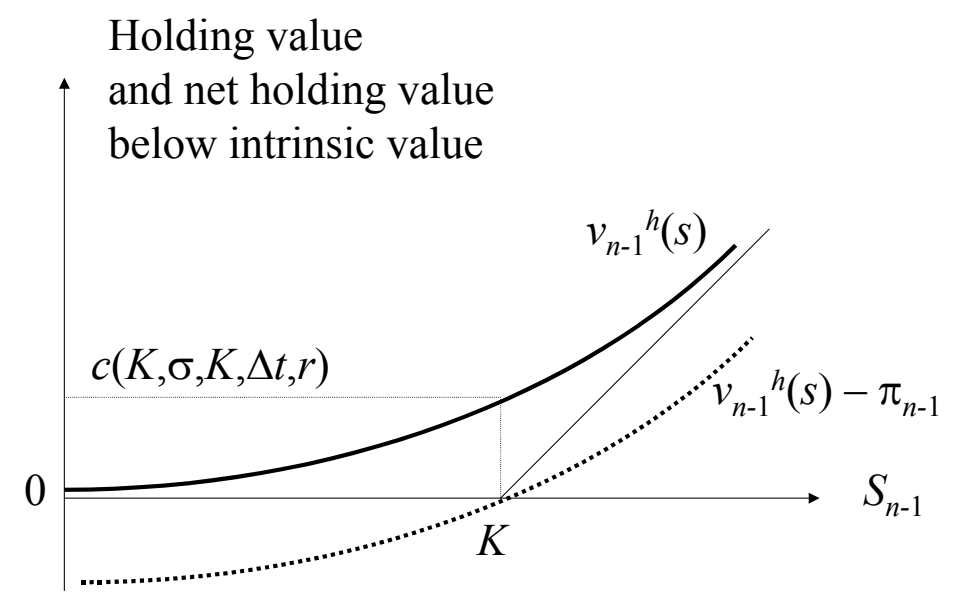

Figure 2

Figure 2 plots the case where the installment is equal to $C(K, \sigma, K, \Delta t, r, \delta)$, which places the net holding value below the intrinsic value. Thus, for all installments greater than $C(K, \sigma, K, \Delta t, r, \delta)$, the holding region vanishes, and the remaining possibilities are exit or exercise.

Corollary 3 Consider an installment vector $\pi=\left(\pi_{1}, \ldots, \pi_{n-1}\right)$ to be paid at $t_{1}, \ldots, t_{n-1}$. If $\pi_{m} \geq C(K, \sigma, K, \Delta t, r, \delta)$, for all $m \in[1, n-1]$, then

$$
v_{0}(s)=C(s, \sigma, K, \Delta t, r, \delta), \quad \text { for all } s>0
$$


Proof. By Proposition 2, we get

$$
\begin{aligned}
v_{0}(s) & =v_{0}^{h}(s) \\
& =E\left[e^{-r \Delta t} v_{1}\left(S_{t_{1}}\right) \mid S_{0}=s\right] \\
& =E\left[e^{-r \Delta t} v^{e}\left(S_{t_{1}}\right) \mid S_{0}=s\right] \\
& =C(s, \sigma, K, \Delta t, r, \delta) .
\end{aligned}
$$

This ends the proof.

\section{$5 \quad$ Numerical experiments}

\subsection{Convergence speed and accuracy}

In the numerical experiments below, we generate the $a_{k}$ 's as the quantiles of $S_{T}$, the distribution of the underlying asset price at maturity, with the addition of the value $K$. The model for the diffusion is the Geometric Brownian Motion with no dividend (Black-Scholes model). Matrices $\left[A_{k i}\right]$ and $\left[B_{k i}\right]$ are precomputed before doing the first iteration.

Table 1 displays the main pricing properties of our approach. First, convergence to the "true" price is fast. A fairly good approximation of the IO price can be obtained almost instantaneously with a 125-point grid. A two-digit accuracy is achieved with a 250-point grid, which involves a computational time of a tenth of a second ${ }^{2}$. A four-digit accuracy can be obtained with a 1000-point grid, which implies a computational time that does not exceed two seconds. Second, note that the number of installments in the contract increase computational time only slightly. For a given grid

\footnotetext{
${ }^{2} \mathrm{CPU}$ times are reported with a $933 \mathrm{MHz}$ Windows $\mathrm{PC}$
} 
size, computational time is divided in two components, a fixed cost to precompute the matrices $A_{k i}$ and $B_{k i}, k=1, \ldots p, i=0, \ldots p$, and a variable cost roughly linear in the number of installments. In particular, computational time increases by around $20 \%$ as the number of installments goes from 0 to 4 . Thus, complex IOs can still be priced with a satisfactory trade-off between accuracy and computational time. Third and most importantly, convergence to the "true" price is monotonic. This allows for extrapolation methods that can significantly reduce computational time for a desired accuracy.

\begin{tabular}{cccccc}
\hline \hline \multirow{2}{*}{$\begin{array}{c}\text { Number of } \\
\text { installments }\end{array}$} & \multicolumn{5}{c}{ Number of grid points } \\
\hline 0 & 125 & 250 & 500 & 1000 & 2000 \\
\hline & $(0.02)$ & $(0.09)$ & $(0.39)$ & $(1.53)$ & $(6.14)$ \\
\hline 1 & 11.49561 & 11.49268 & 11.49236 & 11.49221 & 11.49218 \\
& $(0.02)$ & $(0.11)$ & $(0.41)$ & $(1.61)$ & $(6.45)$ \\
\hline 2 & 9.86059 & 9.85653 & 9.85595 & 9.85575 & 9.85571 \\
& $(0.03)$ & $(0.11)$ & $(0.42)$ & $(1.69)$ & $(6.78)$ \\
\hline 3 & 8.65862 & 8.65312 & 8.65243 & 8.65217 & 8.65211 \\
& $(0.03)$ & $(0.11)$ & $(0.44)$ & $(1.77)$ & $(7.08)$ \\
\hline 4 & 7.80531 & 7.79948 & 7.79856 & 7.79828 & 7.79822 \\
& $(0.03)$ & $(0.11)$ & $(0.47)$ & $(1.84)$ & $(7.39)$ \\
\hline \hline
\end{tabular}

Table 1: IO prices and computational time

Table 1 reports IO upfront payments for various grid sizes with the corresponding CPU time in seconds (in parentheses). The code line is written in $C$ and compiled with GCC. CPU times are obtained with a $933 \mathrm{MHz}$ Windows PC. The IO is a call with equal installments $(\pi=2)$ and the following characteristics: $S=100$, $K=95, \sigma=0.2, r=0.05, \delta=0$, and $T=1$. The number of installments varies 
from 0 to 4 . In the case of zero installment, the call is European and its theoretical price is 13.34647 .

\subsection{Non-redundant IO contracts}

Table 2 reports prices of installment calls for various levels of constant installments. Clearly, these prices are decreasing with the level of installment. They reach the minimum $C(s, \sigma, K, \Delta t, r, 0)$ for installments greater than $\pi=C(K, \sigma, K, \Delta t, r, 0)$, as shown in Proposition 3. For example, when $K=110$, we have

$$
C(100,0.2,110,0.25,0.05,0)=1.191
$$

and

$$
C(110,0.2,110,0.25,0.05,0)=5.076 .
$$

Thus, for any installment greater than 5.076, the holding region vanishes, and the installment call is worth the European call expiring at the next decision date. 


\begin{tabular}{cccc}
\hline \hline Installment & $K=90$ & $K=100$ & $K=110$ \\
\hline 0 & 16.699 & 10.451 & 6.040 \\
0.5 & 15.262 & 9.072 & 4.785 \\
1 & 13.857 & 7.787 & 3.738 \\
1.5 & 12.779 & 6.660 & 2.886 \\
2 & 12.206 & 5.840 & 2.266 \\
2.5 & 11.910 & 5.286 & 1.833 \\
3 & 11.763 & 4.943 & 1.547 \\
3.5 & 11.695 & 4.748 & 1.368 \\
4 & 11.671 & 4.650 & 1.264 \\
4.5 & 11.670 & 4.616 & 1.210 \\
5 & 11.670 & 4.615 & 1.191 \\
5.5 & 11.670 & 4.615 & 1.191 \\
\hline \hline
\end{tabular}

Table 2: IO prices and installment level

Table 2 reports installment call upfront payments for various levels of installment and strikes. Parameters are the following: $S=100, \sigma=0.2, r=0.05$, $\delta=0$, and $T=1$. Exercise rights are quarterly and the IO has three installments.

A direct implication for IO design is that contracts with installments that eliminate the holding region are simply redundant with European options. Within the range of possible installment levels, various hedging properties may be designed. It is worth mentioning that the IO "greeks" may be readily obtained from the approximate value function, a piecewise linear function which is known at all dates for all possible values of the underlying asset. 


\section{Application to ASX installment warrants}

One of the most actively traded installment options throughout the world are currently the installment warrants on Australian stocks. These warrants were launched on the Australian Stock Exchange (ASX) in January 1997. Since then, both the number of listed installment warrants and the trading volume have grown exponentially, as documented by Figure 3 (obtained from the ASX website).

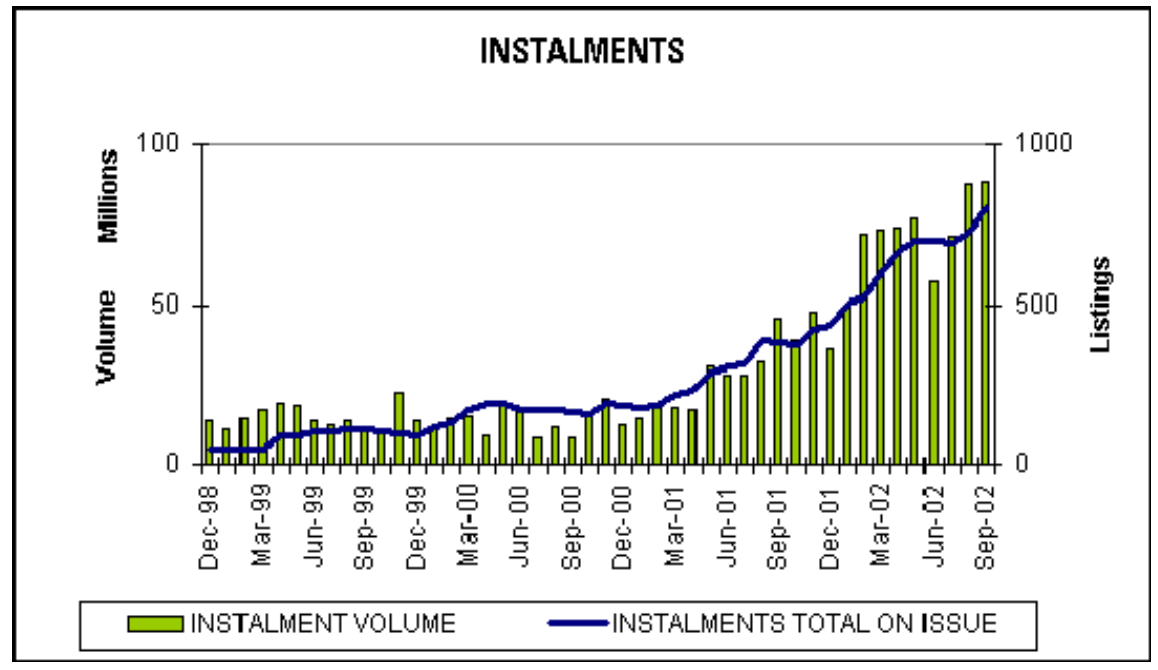

Figure 3: Installment warrants listings and volume

Some of the ASX installment warrants (called rolling installment warrants) have several installments and their expiry date may be up to 10 years. However, most ASX warrants have only one installment with maturities ranging from 1 to 3 years. The single installment is usually set equal to the upfront payment. This clearly puts a restriction on the strike price of the warrant.

In this section, we apply our model to the pricing of installment warrants. By contrast to call options, warrants have a dilution effect on the 
issuer's stocks. Black and Scholes (1973) suggest to price warrants as an option on the issuer's equity (i.e. stocks plus warrants). For so doing, the valuation formula must be adjusted for dilution. Specifically, let $M, N$, and $\gamma$ respectively denote the number of outstanding warrants, the number of outstanding shares, and the conversion ratio. Extending the approach by Lauterbach and Schultz (1990), the installment warrant in this context is interpreted as - a fraction of - an IO issued by the firm. Its payoff process is

$$
Y_{t}=\frac{N \gamma}{N+M \gamma}\left(\frac{V_{t}}{N}-K\right)^{+}, \quad \text { for } t \in\left\{t_{0}=0, \ldots, t_{m}=T\right\},
$$

where $\{V\}=\{N S+M W\}$ is the value of the firm's equity, $\{V / N\}=$ $\{S+M W / N\}$ is the asset underlying the warrant, $\{S\}$ is the stock price of the firm within the warrant life, and $\{W\}$ is the value of the installment warrant.

The DP algorithm described in Section 3 may be easily modified to the pricing of warrants in the context of IOs. The exercise value in (1) is now the payoff of the warrant if exercised optimally

$$
v_{t}^{e}(x)=\frac{N \gamma}{N+M \gamma}(x-K)^{+} .
$$

To compute $W_{0}$, one should solve

$$
v_{0}\left(S_{0}+M W_{0} / N\right)=W_{0} .
$$

This is easy to implement as the procedure gives

$$
v_{0}(x), \text { for all } x>0 \text {. }
$$

As a special case, we get the procedure by Lauterbach and Schultz (1990) for pricing European warrants, namely the price $w$ of the European warrant is obtained using the Black-Scholes formula where: (1) The underlying $S$ 
is replaced with $S+\frac{M}{N} w,(2)$ Volatility $\sigma$ is that of equity returns, and (3) The whole formula is multiplied by the dilution factor $\frac{N \gamma}{N+M \gamma}$. These adjustments result in an equation of the type $w=f(w)$ which must be solved numerically.

Table 3 reports installment warrant prices for degrees of dilution and numbers of installments.

\begin{tabular}{cccccc}
\hline \hline Number of & \multicolumn{5}{c}{ Number of outstanding warrants } \\
installments & $M=0$ & $M=10$ & $M=50$ & $M=100$ & $M=200$ \\
\hline 0 & 13.346 & 13.006 & 11.988 & 11.184 & 10.322 \\
& $(13.346)$ & $(13.006)$ & $(11.989)$ & $(11.185)$ & $(10.324)$ \\
1 & 11.492 & 11.011 & 9.567 & 8.557 & 7.726 \\
2 & 9.855 & 9.364 & 8.054 & 7.289 & 6.790 \\
3 & 8.652 & 8.215 & 7.177 & 6.658 & 6.315 \\
4 & 7.798 & 7.445 & 6.666 & 6.296 & 6.030 \\
\hline \hline
\end{tabular}

Table 3: Installment warrant prices and the dilution effect

Table 3 reports installment warrant upfront payments for various degrees of dilution. The installment warrant has equal installments $(\pi=2)$ and the following characteristics: $S=100, K=95, \sigma=0.2, r=0.05, \delta=0, N=100$, $\gamma=1$, and $T=1$. Grid size is 500 points. The number of outstanding warrants varies from 0 to 200 , and the number of installments varies from 0 to 4 . In the case of zero installment, the warrant is European and its theoretical price (below in parentheses) is given by Lauterbach and Schultz (1990). In the case of $M=0$, the installment warrant is fully diluted and its price equals that of the installment call option (see Table 1).

As can be seen from Table 3, installment warrants prices decrease with the installment and are therefore lower than prices of otherwise identical European warrants. Thus, installment warrants have a weaker dilution effect than European warrants, i.e. the wealth transfer from stockholders to warrantholders is less pronounced. The reason for this is that the presence 
of installments implies that warrants may be abandoned and simply not exercised. As a consequence, the design of installment warrants gives the firm some flexibility in controlling capital dilution when raising funds.

\section{Conclusion}

In this paper, we have developed a pricing methodology for installment options using dynamic programming. This numerical procedure is particularly well suited for IOs because these options are Bermudan-style and involve a limited number of distant exercise dates. Numerical experiments indicate that prices converge monotonically and quickly reach good levels of accuracy.

Our approach is flexible enough to be extended to other pricing issues involving installment options. As an illustration, we show how to adapt our methodology to the pricing of ASX installment warrants. Other extensions might be considered for future research. For instance, levered equity may be seen as a compound call on asset value when debt bears discrete coupons (see Geske (1977)). Consider next that the coupon-bearing debt is callable. At each coupon date, shareholders decide whether or not to call the debt. If they do not call, they decide whether or not to pay the coupon to keep their residual claim on the firm's assets. Consequently, when the firm is financed with defaultable callable debt, levered equity may be priced as an installment call on firm asset value. 


\section{References}

[1] Ben-Ameur, H., M. Breton, and P. L'Écuyer, 2002, "A Dynamic Programming Procedure for Pricing American-style Asian Options", Management Science 48, 625-643.

[2] Billingsley, P., 1995, Probability and Measure, Third Edition, John Wiley and Sons, New York.

[3] Black, F., and M. Scholes, 1973, "The Pricing of Options and Corporate Liabilities", Journal of Political Economy 81, 637-659.

[4] Chen, A. H. Y., 1970, "A Model of Warrant Pricing in a Dynamic Market", Journal of Finance 25, 1041-1059.

[5] Davis, M., W. Schachermayer, and R. Tompkins, 2001, "Pricing, Noarbitrage Bounds and Robust Hedging of Instalment Options", Quantitative Finance 1, 597-610.

[6] Davis, M., W. Schachermayer, and R. Tompkins, 2002, "Instalment Option and Static Hedging", Journal of Risk Finance 3, 46-52.

[7] Davis, M., W. Schachermayer, and R. Tompkins, 2003, "The Evaluation of Venture Capital as an Instalment Option", working paper, University of Frankfurt.

[8] Geske, R., 1977, "The Valuation of Corporate Liabilities as Compound Options“, Journal of Financial and Quantitative Analysis 12, 541-552.

[9] Lauterbach, B., and P. Schultz, 1990, "Pricing Warrants: An Empirical Study of the Black-Scholes Model and Its Alternatives", Journal of Finance 45, 1181-1209. 
[10] Wilmott, P., J. Dewynne, and S. Howison, 1993, Option Pricing: Mathematical Models and Computation, Oxford Financial Press. 